\title{
Ultrasonically guided histological and cytological fine needle biopsies of the pancreas. Reliability and reproducibility of diagnoses
}

\author{
A Glenthøj, M Sehested, S Torp-Pedersen
}

\begin{abstract}
In 100 consecutive patients ultrasonically guided histological and cytological fine needle biopsy specimens were obtained from pancreatic lesions using two different needles with an outer diameter of $0.6 \mathrm{~mm}$. Specimens taken by both cytological and histological fine needle biopsy were examined blindly by two pathologists. When related to the final and reliable diagnosis obtained in 57 patients, the predictive value of a malignant diagnosis was $\mathbf{1 . 0 0}$ for both types of biopsy. The predictive value for a benign diagnosis was $\mathbf{0 . 2 5}$ for histological specimens for both examiners and 0.33 and 0.45 for the two evaluations of the cytological specimens. False benign diagnoses seemed to be related to both sampling error and difficulties in interpreting the biopsy specimens. The intraobserver and interobserver kappa values concerning reproducibility of diagnoses were higher for histological specimens $(0.80$ and $0.74)$ than for cytological specimens $(0.70$ and 0.61). Consistent malignant diagnoses, however, occurred more often with cytological specimens (51 cases) than with histological specimens $(39$ cases $)(p<0.05)$ and consistent diagnoses of insufficient material were more common with histological specimens ( 18 cases $v$ six cases). Cytological fine needle biopsy seems to be the method of choice if only one method is used and a $0.6 \mathrm{~mm}$ needle is used.
\end{abstract}

A reliable preoperative fine needle biopsy diagnosis of a pancreatic lesion is important for both choosing treatment and the prognosis. Cytological fine needle biopsy of the pancreas has been used for many years, ${ }^{1-7}$ but only a few descriptions exist of percutaneous histological fine needle biopsy. ${ }^{8-12}$ The studies on fine needle biopsy of the pancreas have not been conducted as blind morphological studies because clinical information has been an integral part of the microscopic evaluation. Furthermore, reliable final diagnoses other than that from fine needle biopsy are often missing.

The purpose of the present study was to test the unbiased diagnostic capability of both cytological and histological fine needle biopsy to detect malignancy in the pancreas. Furthermore, we wanted to evaluate the reproducibility of diagnoses based on these two techniques in order to elucidate the cause of difficulties in the interpretation of biopsy specimens.

Patients and methods

One hundred consecutive patients in whom ultrasonically guided fine needle biopsies of the pancreas were performed from December 1982 to March 1986 were included in the study. In four patients several biopsies were performed on different occasions. Only the first procedure in these patients was included.

All biopsies were carried out with two $0.6 \mathrm{~mm}$ needles using three passes for each needle. The first needle was a Surecut needle which was used for histological sampling, while the second needle was a Franzén needle which was used for aspiration cytology. The processing of the biopsy specimens has been described. ${ }^{13}$

After coding, both the cytological and histological biopsy specimens were evaluated separately by two pathologists. No clinical information was given except that the specimens were from the pancreas. After six months the biopsies were recoded and re-evaluated by one of the two pathologists.

Both cytological and histological fine needle biopsy specimens were evaluated for (a) adequacy of the material and (b) grading of malignant, suspicion of malignancy, or benign. Comments on tumour type, inflammation, fibrosis, preservation, and amount of material were made.

After the last evaluation the codes were broken and the clinical records were examined in order to establish a final diagnosis. Only histologically proved postmortem diagnoses or surgical biopsies, or both, performed within six months of the fine needle biopsy, were considered as reliable final diagnoses.

Kappa statistics ${ }^{15}{ }^{16}$ were used to estimate the intraobserver and interobserver agreement of diagnoses. A rough estimate of the standard error of kappa was made, even though kappa could not be expected to be normally distributed.

All inconsistent biopsy diagnoses were reevaluated to determine the cause of the inconsistency. Furthermore, cytological and histological specimens were compared to investigate whether consistent diagnoses occurred more frequently with one method.

The time from when a slide was put under the microscope until a diagnosis was reached was registered.

\section{Results}

Biopsy specimens were taken from 41 women and 59 men. The median age of the patients was 65 years (range 28-94 years), 66 years for women and 65 for men. The median follow up time for 89 patients who had died was four months (range 0-38 months). Eleven patients were alive more than 36 months after the biopsy.

\author{
Denmark \\ S Torp-Pedersen \\ Correspondence to: \\ Anders Glenthøj, Department \\ DK-3400 Hillerød, Denmark \\ Accepted for publication \\ 16 October 1989 \\ A Glenth $\varnothing j$ \\ Department of \\ Ultrasound, Herlev
}


TABLE I Comparison between 57 final reliable diagnoses of pancreatic disease and diagnoses by two examiners $(I, I I)$ by cytological and histological fine needle biopsy

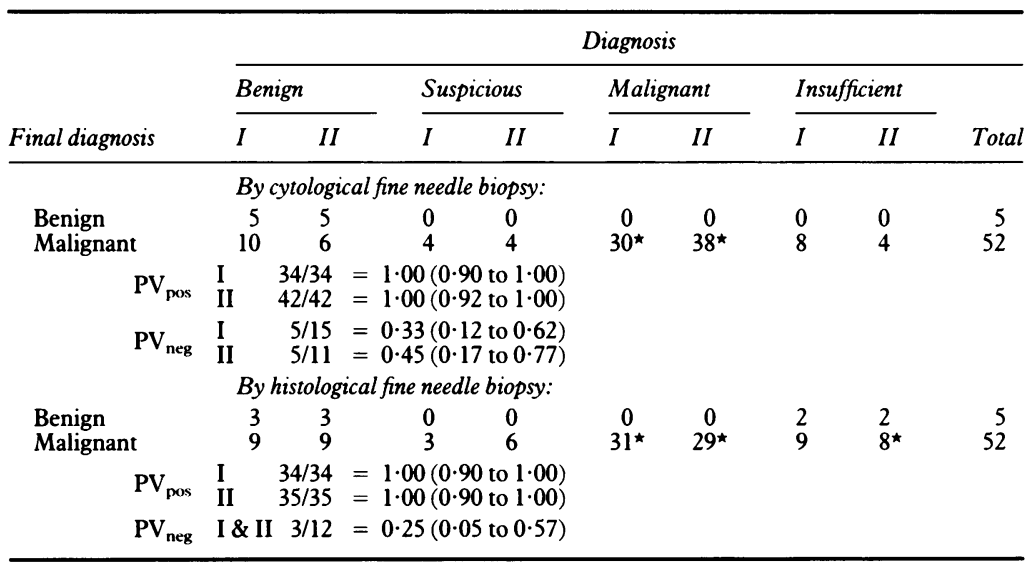
$P V_{\text {pos }}=$ predictive value of a malignant diagnosis. $P V_{\text {neg }}=$ predictive value of a benign diagnosis. The
suspicious diagnoses are considered malignant when the predictive values are calculated. The $95 \%$ suspicious diagnoses are considered malign
confidence interval is shown in parentheses.

^One endocrine tumour.
TABLE II Intraobserver and interobserver reproducibility of diagnosis in 100 cytological fine needle biopsy specimens from the pancreas

\begin{tabular}{lccccr}
\hline (a) Examiner II: & \multicolumn{5}{l}{ First examination } \\
\cline { 2 - 6 } & $B$ & $S$ & $M$ & $I$ & Total \\
\hline Second examination: & & & & & \\
B & 16 & 3 & 3 & & 22 \\
S & 2 & 1 & 3 & & 6 \\
M & 4 & 1 & 59 & & 64 \\
I & 22 & 5 & 65 & 8 & 100 \\
Total & 22 &
\end{tabular}

(b) First examination of examiners I and II

\begin{tabular}{llrrrr} 
& \multicolumn{4}{l}{ Examiner II: } \\
\cline { 3 - 6 } & $B$ & $S$ & $M$ & $I$ & Total \\
\hline Examiner I: & 20 & 2 & 9 & 1 & 32 \\
B & & 1 & 5 & & 6 \\
S & 2 & 2 & 49 & & 49 \\
I & 22 & 5 & 65 & 8 & 100 \\
Total & Kappa $=0.61(\varepsilon=0.064)$ & & \\
&
\end{tabular}

Final and reliable diagnoses were obtained for 57 patients (necropsy 27, surgical biopsies or surgical specimens 30 ). Some of the surgically verified cases were reconfirmed at necropsy. In seven patients necropsy was performed more than six months after the biopsy, and in one patient a surgical biopsy was performed after six months. Twenty seven patients who died had had no surgery or necropsy; 25 of these died with signs of pancreatic disease, while two patients died of causes probably unrelated to pancreatic disease. Eight patients without reliable final diagnoses were alive roughly three years after the fine needle biopsy.

The 57 final reliable diagnoses were as follows: pancreatitis (acute or chronic) five, endocrine pancreatic tumour one, infiltration of the pancreas from a cancer of the common bile duct or papilla of Vater two, pancreatic adenocarcinoma (including one adenosquamous carcinoma) 49. Only three of the 57 patients were alive three years after the biopsy (one cancer of the papilla of Vater, one pancreatic adenocarcinoma, and one pancreatitis).

In Table I the results of the blind cytological and histological examinations are compared to the 57 reliable final diagnoses. None of the examiners reached a false diagnosis of malignancy for the five benign cases, all of whom had pancreatitis. Inflammation was diagnosed cytologically in these five cases whereas it was found histologically in only two of five cases. Of the remaining three histological specimens from benign lesions, two were judged insufficient and one showed normal pancreatic tissue. The predictive values of a malignant or benign diagnosis are shown in Table I. If the eight patients who had no reliable final diagnosis - but were alive three years after the biopsy - are considered to have a benign disease the predictive values for a benign diagnosis increased to 0.49 (examiner I) and $\mathbf{0 . 5 7}$ (examiner II) for cytological diagnosis and to 0.50 for histological diagnosis (both examiners).

Among the false benign diagnoses inflammation or fibrosis was diagnosed by both cytology and histology in about $50 \%$ of the cases while the remaining diagnoses were normal
$\mathrm{B}=$ benign $; \mathrm{S}=$ suspicion of malignancy; $\mathrm{M}=$ malignant;

$\mathrm{I}=$ insufficient material. $\varepsilon=$ estimated standard error.

pancreatic cells or tissue, except for three histological specimens where only normal liver tissue was found by both examiners. The single endocrine tumour was correctly diagnosed by both examiners by cytology and by examiner I by histology. Examiner II found the histological specimen from that tumour insufficient due to crushing artefacts.

The intraobserver and interobserver reproducibility of cytological and histological diagnoses are shown in Tables II and III. The kappa values were somewhat higher for histology $(0 \cdot 80$ and 0.74$)$ than for cytology $(0.70$ and 0.61$)$.

The total number of consistent and inconsistent diagnoses are given in Table IV. Consistent diagnoses of insufficient material were more common by histology (18 cases) than by cytology (six cases). The most likely explanations for inconsistent diagnoses are given in Table IV. At re-evaluation the most common explanation for inconsistent diagnoses was that the biopsy specimen were of poor quality.

TABLE III Intraobserver and interobserver reproducibility of diagnosis in 100 histological fine needle biopsy specimens from the pancreas

\begin{tabular}{lrrrrr}
\hline (a) Examiner II: & \multicolumn{5}{l}{ First examination } \\
\cline { 2 - 6 } & $B$ & $S$ & $M$ & $I$ & Total \\
\hline Second examination: & & & & & \\
B & 23 & 1 & 1 & 1 & 26 \\
S & 1 & 5 & 2 & & 8 \\
M & 1 & 3 & 39 & 19 & 43 \\
I & 1 & 2 & 1 & 19 & 23 \\
Total & 26 & 11 & 43 & 20 & 100 \\
& Kappa $=0.80(\varepsilon=0.049)$ & &
\end{tabular}

(b) First examination of examiners $I$ and $I I$

Examiner II:

\begin{tabular}{lrrrrr} 
& $B$ & $S$ & $M$ & $I$ & Total \\
\cline { 2 - 6 } Examiner I: & & & & & \\
B & 21 & 1 & 2 & & 24 \\
S & 1 & 3 & 2 & 1 & 6 \\
M & 3 & 5 & 39 & 19 & 48 \\
I & 1 & 2 & & 19 & 22 \\
& 26 & 11 & 43 & 20 & 100 \\
& Kappa $=0 \cdot 74(\varepsilon=0.055)$ & & \\
\hline
\end{tabular}

$\mathrm{B}=$ benign; $\mathrm{S}=$ suspicion of malignancy; $\mathrm{M}=$ malignant $\mathrm{I}=$ insufficient material. $\varepsilon=$ estimated standard error. 
TABLE IV Consistency of 100 cytological and 100 histological fine needle biopsy specimens from the pancreas. Consistency is defined as total agreement between three examinations by two examiners. Possible causes of inconsistency were evaluated by re-examination

\begin{tabular}{lr}
\hline Cytological fine needle biopsy: & \\
Consistency & \\
Malignant & 51 \\
Suspicious & 1 \\
Benign & 14 \\
Insufficient & 6 \\
Total & 72 \\
Inconsistency re-evaluation & \\
Very few malignant cells & 13 \\
Discrimination between malignant cells and & 10 \\
inflammatory changes difficult & 1 \\
Preparation too thick & 4 \\
Difficult to interpret (no inflammation) & 28 \\
Total & \\
Histological fine needle biopsy: & 39 \\
Consistency & 2 \\
Malignant & 19 \\
Suspicious & 18 \\
Benign & 78 \\
Insufficient & \\
Total & 12 \\
Inconsistency re-evaluation & 6 \\
Biopsy too small & 1 \\
Traumatised biopsy & 3 \\
Necrotic tissue & 22 \\
Fibrosis with too little epithelial tissue & \\
Total & \\
\hline
\end{tabular}

Table $\mathrm{V}$ shows that in 51 cases a malignant cytological diagnosis was reached in all three evaluations, while in 39 cases a consistent malignant histological diagnosis was reached. This difference is significant $(\mathrm{p}<0.05)$.

The two examiners spent 11 and eight minutes respectively evaluating the slides from cytological biopsies when a benign diagnosis was given, while a malignant diagnosis took four and three minutes respectively (median values). For histological biopsies four and two minutes were taken to make a benign diagnosis, but both examiners reached a malignant diagnosis in two minutes (median values). A malignant diagnosis that could not be reproduced took approximately twice as long to evaluate as a malignant diagnosis which could be reproduced.

\section{Discussion}

Only 11 of our 100 patients were alive three years after the biopsy. This can partly be explained by the fact that the majority of our patients had a pancreatic adenocarcinoma. At least four of the patients with pancreatitis, however, died within three years of the biopsy, making survival data unreliable in distinguishing between benign and malignant pancreatic disease.

Modern imaging techniques can show a pancreatic lesion, but the images do not discriminate between inflammatory and malignant disease,

TABLE V Comparison between 100 cytological and 100 histological fine needle biopsy diagnoses from the pancreas

\begin{tabular}{lrcr}
\hline & \multicolumn{3}{c}{ Histological fine needle biopsy } \\
\cline { 2 - 4 } & $M \times 3$ & Not $M \times 3$ & Total \\
\hline Cytological fine needle biopsy: & 31 & 20 & 51 \\
$M \times 3$ & 8 & 41 & 49 \\
Not $M \times 3$ & 39 & 61 & 100 \\
Total & & & \\
\hline
\end{tabular}

Cytological biopsy results more frequently in a consistent malignant diagnosis than histological biopsy ( $p<0 \cdot 05$, sign test) $\mathbf{M} \times \mathbf{3}=$ malignant in all three examinations.

Not $M \times 3=$ other diagnoses or inconsistent diagnoses. making further characterisation desirable. Percutaneous cytological fine needle biopsy of the pancreas is considered a reliable low risk procedure. ${ }^{1-46717-24}$ The specificity of this type of biopsy of pancreatic lesions is usually reported to be $1 \cdot 00$, but in some investigations false malignant diagnoses occur. ${ }^{2526}$ The sensitivity is around 0.80 in most reports, ${ }^{27}$ but as high as 0.97 in reports where the method of verification is somewhat doubtful. ${ }^{78}$ Since most investigations have a low proportion of benign cases the predictive values seem to be more useful when describing the reliability of cytological fine needle biopsy. The predictive value of a benign diagnosis of disease of the pancreas by this method has been found to be as low as $0 \cdot 23 .{ }^{26}$

The present cytological investigation, which, unlike the studies mentioned above, was conducted blindly, confirms that a malignant diagnosis by cytological fine needle biopsy is reliable since no false malignant diagnosis occurred among the five patients with verified benign disease (Table I). We did not, however, find a benign diagnosis by this method reliable since the predictive values for that diagnosis achieved by the two examiners were only 0.33 and 0.45 respectively. The low predictive value of a benign diagnosis could partly be explained by sampling error since many carcinomas are surrounded by an inflammatory reaction. ${ }^{28}$ Our study on intraobserver reproducibility (Table IIa), however, shows that in seven cases a shift between a benign and a malignant diagnosis occurred. Difficulties in interpreting the slides may therefore be part of the reason for the low predictive value of a benign diagnosis by cytological biopsy. This was confirmed by re-evaluation (Table IV), which showed that both a low cellularity and difficulty in interpreting cellular material were the most likely causes of inconsistent diagnoses by cytological biopsy.

Histological fine needle biopsy provides the morphological benefits concerning structure known from surgical biopsies. A few studies on this type of biopsy including some pancreatic tumours have been published. ${ }^{8912}$ It was our hope that histological biopsy would provide more reliable and reproducible diagnoses than cytological biopsy. The predictive values, however, of both a malignant and a benign diagnosis using this were similar to those achieved by cytological biopsy (Table I). Sampling error was obvious in the three histological biopsy specimens where only liver tissue was found. These cases represent contamination from a transhepatic puncture route.

The kappa values for intraobserver and interobserver reproducibility were somewhat higher for histological biopsy (Table III) than for cytological biopsy (Table II), but much lower than the kappa values we have achieved in liver biopsy. ${ }^{29}$ It should be noted that any disagreement was given the same weight when the kappa values were calculated. The fact that a consistent diagnosis of insufficient material occurred more frequently with histological than with cytological specimens largely explains the differences in kappa values. A better quality of histological biopsy specimen is desirable (Table IV) since inconsistent diagnoses could be related 
to the size and the technical quality of the biopsy specimen. We have now changed the needle diameter from 0.6 to $0.8 \mathrm{~mm}$ to increase the sensitivity of a malignant diagnosis of pancreatic lesions, even though the risk of causing pancreatitis might thereby be increased. ${ }^{21}$ Factors other than the diameter of the needle, such as the skill of the ultrasonographer and the texture of the punctured lesions, are also important for obtaining an adequate biopsy. It is difficult to obtain a tissue core from a very soft or necrotic tumour. If peripheral parts of a lesion are biopsied the biopsy specimen may represent a chronic or acute pancreatitis secondary to a centrally located carcinoma.

Examiner I was more cautious than examiner II when making a diagnosis by cytological biopsy (Tables Ia and IIb), but otherwise there were no obvious differences between the two examiners. The similar diagnostic abilities of the examiners are also reflected in the small differences between intraobserver and interobserver kappa values (Tables II and III). Like Hadju et al, ${ }^{7}$ we believe that the skill of the examiner is of importance, but difficulties in achieving better results seem to be related more to the quality of the biopsy once a certain routine is established.

Cytological fine needle biopsy is more sensitive in making a consistent malignant diagnosis than histological fine needle biopsy (Table V). The combined use of both methods is of value since histological biopsy specimens provided eight consistent malignant diagnoses in cases where cytological biopsy specimens were not consistently malignant (Table V). If only one method is used, however, and a $0.6 \mathrm{~mm}$ needle is used, cytological fine needle biopsy is the method of choice because of its sensitivity and because it is cheaper and faster to process.

1 Hancke S, Holm HH, Kock F. Ultrasonically guided fine needle biopsy of the pancreas. Surg Gynecol Obstet 1975; 140 : needle $361-3$.

2 Holm HH, Torp-Pedersen S, Larsen T, Juul N. Percutaneous fine needle biopsy. Clin Gastroenterol 1985; 14: 423-49.

3 Droese $\mathrm{M}$, Altmannsberger $\mathrm{M}$, Kehl $\mathrm{A}$, et al. Ultrasoundguided percutaneous fine needle aspiration biopsy of abdominal and retroperitoneal masses. Accuracy of cytology in the diagnosis of malignancy, cytologic tumor typing and use of antibodies to intermediate filaments in selected cases. Acta Cytol 1984; 28: 368-84.

4 Alpern GM, Dekker A. Fine needle aspiration of the pancreas. Acta Cytol 1985; 29: 873-8.

5 Taavitsainen M, Koivuniemi A, Bondestam S, Kivisaari L, Tierala E. Ultrasonically guided fine-needle aspiration biopsy in focal pancreatic lesions. Acta Radiol 1987; 28: biopsy in
6 Teplick SK, Haskin PM, Kline TS, Sammon JK, Laffey PA. Percutaneous pancreaticobiliary biopsies in 173 patients using primarily ultrasound or fluoroscopic guidance. Cardiovasc Intervent Radiol 1988; 11: 26-8.

7 Hajdu EO, Kumari-Subaiya S, Phillips G. Ultrasonically guided percutaneous aspiration biopsy of the pancreas. Semin Diagn Pathol 1986; 3: 166-75.

8 Wittenberg J, Mueller PR, Ferrucci JT, et al. Percutaneous core biopsy of abdominal tumors using 22 gauge needles. Further observations. AfR 1982; 139: 75-80.

9 Lieberman RP, Hafez GR, Crummy AB. Histology from aspiration biopsy: Turner needle experience. $A \mathcal{F R} 1982$; 138: $561-4$.

10 Holm HH, Glenthøj A, Torp-Pedersen S. Ultrasonicallyguided biopsy of abdominal and retroperitoneal lesions. Semin Intervent Radiol 1986; 3: 271-82.

11 Chagnon S, Cochand-Priollet B, Jacquenod P, Vilgrain V, Blery $M$. Intérêt de la cytoponction associée à la microbiopsie dans les masses solides du pancréas. $\mathcal{F}$ Radiol 1987; 68: $733-6$.

12 Yamamoto R, Tatsuta M, Noguchi S, et al. Histocytologic diagnosis of pancreatic cancer by percutaneous aspiration biopsy under ultrasonic guidance. Am $\mathcal{F}$ Clin Pathol 1985; 83: 409-14.

13 Torp-Pedersen S, Juul N, Vyberg $M$. Histological sampling with a 23 gauge modified Menghini needle. $\mathrm{Br} \mathcal{F}$ Radiol 1984; 57: $151-4$.

14 Glenthøj A, Sehested M, Torp-Pedersen S. Reliability of histological and cytological fine needle biopsies from focal liver lesions. Histopathology 1989; 15: 375-83.

15 Cohen J. Weighted kappa: nominal scale agreement with provision for scaled disagreement or partial credit. Psychol Bull 1968; 70: 213-20.

16 Fleiss JL, Cohen J, Everitt BS. Large sample standard errors of kappa and weighted kappa. Psychol Bull 1969; 22: 323-7.

17 Tao L, Ho C, McLoughlin MJ, McHattie J. Percutaneous aspiration biopsy of the pancreas. Cytodiagnosis of pancreatic carcinoma. Acta Cytol 1978; 22: 215-20.

18 Dinkov L, Donov M, Brailski. Feinnadelaspirationszytologie der Leber- und Pankreastumoren. Arch Geschwulstforsch 1986; 56: 357-65.

19 Bohár L, Bajtai A, Sarkadi G, Erös A, Gönczi J. Percutaneous aspiration biopsy of the pancreas under ultrasonic guidance. Radiol Diagn 1985; 26: 683-7.

20 Celle G, Savarino V, Biggi E, et al. Fine-needle aspiration cytodiagnosis: a simple and safe procedure for cancer of the cytodiagnosis: a simple and safe procedure for cance

21 Mueller PR, Miketic LM, Simeone JF, et al. Severe acute pancreatitis after percutaneous biopsy of the pancreas. $A \mathcal{F} R$ $1988 ; 151: 493-4$.

22 Frölich E, Wehrmann K, Seeliger H, Vierling P, Frühmorgen $P$. Ultraschallgezielte Feinnadelzytologie und Feinnadelhistologie bei umschriebenen Pankreasprozessen. Leber Magen Darm 1988; 18: 236-44

23 Binder T, Swobodnik W, Wechsler JG, et al. Sonographisch geführte Fein- und Grobnadelpunktion im abdominalen
und retroperitonealen Raum. Dtsch Med Wochenschr 1988; 113: 43-8.

24 Oland J, Rosen A, Reif R, Sayfan J, Kent V, Orda R. Fine needle aspiration cytology of the pancreas. $\mathcal{F}$ Surg Oncol 1988; 38: 14-5.

25 Waldner B, Dorval ED, Anthonioz P, et al. Cytoponction pancréatique à l'auguille fine. A propos de 45 observations. pancréatique à l'auguille fine. A propos

26 Mitchell ML, Bittner CA, Wills JS, Parker FP. Fine needle aspiration cytology of the pancreas. Acta Cytol 1988; 32: 447-51.

27 Bret PM, Nicolet V, Labadie M. Percutaneous fine-needle aspiration biopsy of the pancreas. Diagn Cytopathol 1986; 2: 221-7.

28 Klöppel G. Pancreatic, non-endocrine tumours. In: Klöppel G, Heitz PU, eds. Pancreatic pathology. Edinburgh: Churchill Livingstone, 1984: 79-113.

29 Glenthøj A, Sehested M. Histological and cytological fine needle biopsies from focal liver lesions. Intra- and interobserver reproducibility of diagnoses. APMIS 1989; 97: 611-8. 